\section{Kann Cholelithiasis Dünndarmkrebs auslösen?}

\author{
Mit einer Cholelithiasis steigt nicht nur das Risiko für maligne Erkrankungen \\ der Gallenblase und des Gallengangs.
}

\begin{abstract}
A uch die seltenen Karzinome des Dünndarms treten deutlich häufiger auf als in Abwesenheit von Gallensteinleiden, wie eine in den Annals of Oncology publizierte Untersuchung belegt. Dabei handelt es sich um die retrospektive Auswertung einer Serie von Fall-Kontroll-Studien zu verschiedenen Neoplasien, die von 1982 bis 2009 in Italien und der Schweiz durchgeführt wurden.

Von 23 Patienten mit einem Karzinom des Dünndarms hatten fünf eine Cholelithiasis in der Anamnese, bei den Kontrollpatienten war das nur bei 20 von 230 der Fall. Damit bestand bei den SteinPatienten mit einer Odds Ratio von 3,96 ein signifikant erhöhtes Erkrankungsrisiko. Etwas geringer, aber immer noch signifikant war der Risikoanstieg, wenn diese Studie mit drei älteren Studien zum Dünndarmkrebs in einer Metaanalyse zusammengefasst wurde: Beruhend auf 72 Karzinomen in Kombination mit einer Cholelithiasis wurde eine Odds Ratio von 2,35 errechnet.
\end{abstract}

In den Fall-Kontroll-Studien wurde bei Patienten mit Gallensteinleiden auch eine leicht erhöhte Rate von Prostata- und Nierenzellkrebs festgestellt (OR 1,36 und 1,57). Möglicherweise spiegeln diese Zahlen aber nur gemeinsame Risikofaktoren wieder: Hohe Cholesterinwerte können Prostatakarzinome begünstigen, Übergewicht und seine Folgeerkrankungen zur Entstehung von Nierenzellkarzinomen beitragen.

Andere Krebsarten scheinen durch das Steinleiden nicht beeinflusst zu werden, wie die Analyse der übrigen Fall-KontrollStudien ergab. Die untersuchten Fälle umfassten hunderte von Karzinomen von Oropharynx, Ösophagus, Magen, Kolorektum, Leber, Pankreas, Larynx, Brust, Endometrium, Ovarien und Blase.

Fazit: Die Befunde zusammen mit der bevorzugten Lokalisation von Adenokarzinomen des Dünndarms - im Duodenum nahe der Ampulla vateri - sprechen dafür, dass die Gallenflüssigkeit dort kar-

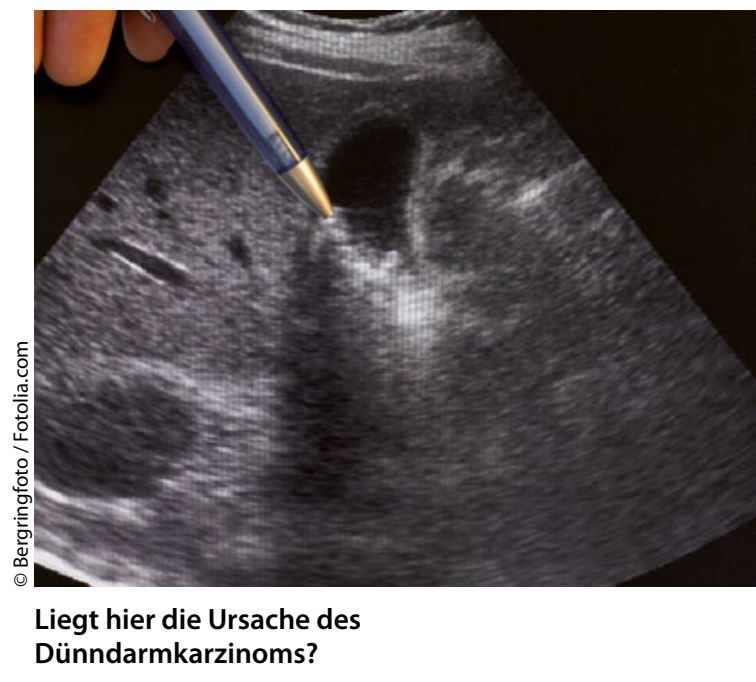

zinogene Wirkungen entfalten kann. Bereits in einer früheren Studie gab es Hinweise, dass Bestandteile der Galle genotoxisch wirken und über eine lokale Zellschädigung die mitotische Aktivität im angegriffenen Gewebe erhöhen können.

Beate Schumacher

Tavani A et al. History of cholelithiasis and cancer risk in a network of case-control studies. Ann Oncol. 2012; DOI: 10.1093/annonc/ mdr581

Allerdings wurden bei sozial Schwächeren nur Karzinome des Rektums und des linken Kolons vermehrt diagnostiziert. In Bezug auf proximale Karzinome erwiesen sich die verschiedenen Gesellschaftsschichten als gleichgestellt.

Fazit: Bei sozial deprivierten Menschen treten häufiger Tumoren des Rektums und des linken, nicht aber des rechten Kolons auf. Möglicherweise sind Unterschiede in der Karzinogenese für die lokale Differenz verantwortlich, so die Vermutung der Studienautoren. Auch die geringere Inanspruchnahme der Früherkennung bei sozial unterprivilegierten Gruppen könne dazu beitragen, weil bei der Koloskopie vor allem Tumoren des Rektums und des linken Kolons entdeckt werden.

Beate Schumacher Fälle wurden anhand eines Tumorregisters festgestellt.

Im Beobachtungszeitraum wurden bei insgesamt 7.676 Studienteilnehmern primäre invasive kolorektale Adenokarzi- nome diagnostiziert, davon bei $46,6 \%$ im rechten und bei 26,7\% im linken Kolon sowie bei $25,9 \%$ im Rektum (bei 0,8\% fehlte eine Angabe). Die KRK-Inzidenz war umso höher, je geringer der Bildungsgrad der Teilnehmer und je weniger privilegiert ihre Nachbarschaft war. Wer weniger als zwölf Jahre eine Schule besucht hatte, dessen KRK-Risiko lag (nach Adjustierung anderer Risikofaktoren) um 19\% höher als das von Personen mit einem akademischen Titel. Eine verarmte Nachbarschaft erhöhte das KRK-Risiko im Vergleich zu einem Luxusviertel um $16 \%$.
Chyke A et al. Socioeconomic status and the risk of colorectal cancer.

Cancer 2012; DOI: 10.1002/cncr.26677. 